\title{
Simplification of a System of Geodesic Equations by Reference to Conservation Laws
}

\author{
Uchechukwu Opara \\ Correspondence: Veritas University (VUNA), Abuja, Nigeria \\ Received: August 2, 2020 Accepted: September 3, 2020 Online Published: September 10, 2020 \\ doi:10.5539/jmr.v12n5p37 \\ URL: https://doi.org/10.5539/jmr.v12n5p37
}

\begin{abstract}
This paper is purposed to exploit prevalent premises for determining analytical solutions to differential equations formulated from the calculus of variations. We realize this premises from the statement of Emmy Noether's theorem; that every system in which a conservation law is observed also admits a symmetry of invariance (Olver, 1993, pp.242; Dresner, 1999, pp.60-62 ). As an illustration, the infinitesimal symmetries for Ordinary Differential Equations (O.D.E's) of geodesics of the glome are explicitly computed and engaged following identification of a relevant conservation law in action. Further prospects for analysis of this concept over the same manifold are then presented summarily in conclusion.
\end{abstract}

Keywords: glome, geodesics, calculus of variations, conservation Laws, systems of differential equations, infinitesimal symmetries

\section{Introduction}

The 3-sphere, otherwise termed the glome, is a Riemannian manifold at the center of several revolutionary conjectures and advancements in modern mathematical theory. As prominent examples, one may consider the famous Poincaré conjecture and the Ricci Flow theorem of Hamilton on closed 3-dimensional manifolds with everywhere positive scalar curvature (Cao et al., 2003, pp.128). The former example challenges an interested mind on meticulous details of differential topology, while the latter is a relatively modern sprout of Pseudo-Riemannian geometry requiring assorted topological and analytical tools. It may be argued that Hamilton's theorem is conveniently suited towards verification of the Poincaré conjecture from an assortment of partial vantage points.

In a prior pre-print publication by the author of this article (Opara, 2017), significant reference is made to the formulation of the geodesic differential equations studied in this article. The requisite techniques harnessed from the calculus of variations in the author's prior arXiV publication cover issues such as well-posedness and determination of integral curves of the formulated system of non-linear differential equations via suitable coordinate systems.

In this study however, the focus shifts to an aspect of the computational wealth available to be harnessed from conservation laws in action during the course of analogous physical processes on the glome. Since the choice manifold is of intermediate dimension, the computational work included may be readily confirmed manually, or it could perhaps motivate an exploration of digital software for similar problems. An advantage of the choice of manifold here is that for many coordinate systems used to capture it, the associated formulated equations have solutions in terms of elementary functions. Moreover, the practical scientific essence of the choice manifold cannot be discarded, as several formulations from the Ricci Flow are formally analogous to heat flow along manifolds and some formulations are adapted to the analysis required in modern cosmological mechanics (Cao et al., 2003, pp. 95, 107).

In order to come to terms with a conservation law in play in the course of traversing geodesics of a smooth manifold by a particle without slipping, one may consult the weak formulation process from the stage of differentiation in Banach path spaces. Where the geodesic curve computed in the domain of a relevant coordinate system is parametrized by $t$, we may recall that all such curves satisfy

$$
\Lambda_{u_{i}}-\frac{d}{d t}\left(\Lambda_{\dot{u}_{i}}\right)=0
$$

given the arclength functional

$$
s_{I, t}=\int_{I} \Lambda\left(t, u_{1}(t), u_{2}(t), \cdots, u_{n-1}(t), \dot{u_{1}}, \dot{u}_{2}, \cdots, \dot{u}_{n-1}\right) d t
$$

for an analytical hypersurface embedded in $\mathbb{R}^{n}$ and spatial parameters $u_{i}: 1 \leq i \leq n-1$ (Brunt, 2004, pp.33). Conservation of the quantity $\left[\Lambda_{u_{i}}-\frac{d}{d t}\left(\Lambda_{u_{i}}\right)\right]$ mentioned above seems to bear analogy to a phenomenon in classical mechanics independent of gravitational influence. 


\section{Method of Formulation}

The procedure for computing infinitesimal symmetries accommodated by the system of Euler-Lagrange equations is detailed rather succinctly in the following two theorems.

Theorem 1 (Olver, 1993, pp.253) A connected group of transformations $G$ acting on $M$ is a variational symmetry group of the functional $s_{I, t}$ if and only if

$$
p r^{(j)} \mathbf{v}(\Lambda)+\Lambda \frac{d \xi}{d t}=0,
$$

for all $\left(t, u^{(j)}\right) \in M$ and every infinitesimal generator

$$
\mathbf{v}=\xi(t, u) \frac{\partial}{\partial t}+\sum_{\alpha=1}^{q} \phi_{\alpha}(t, u) \frac{\partial}{\partial u^{\alpha}}
$$

of $G$.

Theorem 2 (Olver, 1993, pp.255) If $G$ is a variational symmetry group of the functional $s_{I, t}=\int_{I} \Lambda(t, u) d t$, then $G$ is a symmetry group of the associated Euler-Lagrange equations.

Consider the requisite Lagrangian computed in a prior publication by the author (Opara, 2017, pp.10) for discovering equations of geodesics for the glome:

$$
s_{I, u_{1}}=\int_{I} \sqrt{1+\cos ^{2} u_{1}\left(\frac{d u_{2}}{d u_{1}}\right)^{2}+\cos ^{2} u_{1} \cos ^{2} u_{2}\left(\frac{d u_{3}}{d u_{1}}\right)^{2}} d u_{1}:=\int_{I} \Lambda d u_{1}
$$

for an appropriate interval $I \subseteq\left[\frac{-\pi}{2}, \frac{\pi}{2}\right]$. We hereby reckon with the hyperspherical co-ordinate system,

$$
\begin{aligned}
& f:\left[\frac{-\pi}{2}, \frac{\pi}{2}\right]^{2} \times[0,2 \pi] \quad \longrightarrow \quad S^{3} \subset \mathbb{R}^{4} \\
& \left(u_{1}, u_{2}, u_{3}\right) \quad \longmapsto f\left(u_{1}, u_{2}, u_{3}\right)=\left(x_{1}, x_{2}, x_{3}, x_{4}\right)
\end{aligned}
$$

$f\left(u_{1}, u_{2}, u_{3}\right)=\left(\cos u_{1} \cos u_{2} \cos u_{3}, \cos u_{1} \cos u_{2} \sin u_{3}, \cos u_{1} \sin u_{2}, \sin u_{1}\right)$.

For this study, we shall identify with the following renaming of variables:

$$
\left(s_{I, u_{1}}, u_{1}, u_{2}, u_{3}\right):=(\mathcal{L}, x, y, v) .
$$

Assuming that $\mathcal{L}$ accommodates an infinitesimal symmetry

$$
\mathbf{v}=\xi \frac{\partial}{\partial x}+\phi \frac{\partial}{\partial y}+\eta \frac{\partial}{\partial v}
$$

we must equivalently have the following from Theorem 1 above:

$$
p r^{(1)} \mathbf{v}(\Lambda)+\Lambda \frac{d \xi}{d x}=0 .
$$

We have taken $j=1$ in the statement of the theorem, because no derivative in $\Lambda$ is higher than the first. Now, we reckon that $p r^{(1)} \mathbf{v}=\mathbf{v}+\phi^{x} \frac{\partial}{\partial y_{x}}+\eta^{x} \frac{\partial}{\partial v_{x}}$, where

$$
\begin{aligned}
& \begin{aligned}
& \phi^{x}=\mathcal{D}_{x}\left(\phi-\xi y_{x}\right)+\xi y_{x x} \\
&=\phi_{x}+\phi_{y} y_{x}+\phi_{v} v_{x}-\left[\xi_{x} y_{x}+\xi_{y}\left(y_{x}\right)^{2}+\xi_{v} v_{x} y_{x}\right], \\
&=\mathcal{D}_{x}\left(\eta-\xi v_{x}\right)+\xi v_{x x} \\
&=\eta_{x}+\eta_{y} y_{x}+\eta_{v} v_{x}-\left[\xi_{x} v_{x}+\xi_{y} y_{x} v_{x}+\xi_{v}\left(v_{x}\right)^{2}\right] . \\
& \xi \frac{\partial}{\partial x}+\phi \frac{\partial}{\partial y}+\eta \frac{\partial}{\partial v}+
\end{aligned} \\
& \operatorname{pr}^{(1)} \mathbf{v}(\Lambda)=\left(\begin{array}{c}
\phi_{x}+\phi_{y} y_{x}+\phi_{v} v_{x}-\left[\xi_{x} y_{x}+\xi_{y}\left(y_{x}\right)^{2}+\xi_{v} v_{x} y_{x}\right]+ \\
\eta_{x}+\eta_{y} y_{x}+\eta_{v} v_{x}-\left[\xi_{x} v_{x}+\xi_{y} y_{x} v_{x}+\xi_{v}\left(v_{x}\right)^{2}\right]
\end{array}\right)(\Lambda) \\
& =\frac{\xi}{2 \Lambda}\left(-2(\cos x \sin x) y_{x}^{2}-2\left(\cos x \sin x \cos ^{2} y\right) v_{x}^{2}\right)+\frac{\phi}{2 \Lambda}\left(-2\left(\cos ^{2} x \cos y \sin y\right) v_{x}^{2}\right) \\
& +\frac{1}{2 \Lambda}\left(\phi_{x}+\phi_{y} y_{x}+\phi_{v} v_{x}-\left[\xi_{x}+\xi_{y} y_{x}+\xi_{v} v_{x}\right] y_{x}\right)\left(\cos ^{2} x\right) \cdot 2 y_{x} \\
& +\frac{1}{2 \Lambda}\left(\eta_{x}+\eta_{y} y_{x}+\eta_{v} v_{x}-\left[\xi_{x}+\xi_{y} y_{x}+\xi_{v} v_{x}\right] v_{x}\right)\left(\cos ^{2} x \cos ^{2} y\right) \cdot 2 v_{x} .
\end{aligned}
$$




\section{Computational Results}

For fulfillment of admittance of the infinitesimal symmetry criterion derived above from Theorem 1, we must solve the equation:

$$
\Lambda \cdot p r^{(1)} \mathbf{v}(\Lambda)+\Lambda^{2} \cdot\left(\xi_{x}+\xi_{y} y_{x}+\xi_{v} v_{x}\right)=0 .
$$

We hence evaluate the coefficients of the various uneliminated monomials involved in this equation to zero, as obtained in the table below.

\begin{tabular}{c} 
MONOMIAL \\
\hline 1 \\
$y_{x}$ \\
$v_{x}$ \\
$\left(y_{x}\right)^{2}$ \\
$\left(v_{x}\right)^{2}$ \\
$y_{x} v_{x}$
\end{tabular}

$\frac{\text { COEFFICIENT }}{\xi_{x}=0}$
$\phi_{x} \cos ^{2} x+\xi_{y}=0$
$\eta_{x} \cos ^{2} y \cos ^{2} x+\xi_{v}=0$
$-\xi \cos x \sin x+\phi_{y} \cos ^{2} x=0$
$-\xi \sin x \cos y-\phi \cos x \sin y+\eta_{v} \cos x \cos y=0$
$\eta_{y} \cos ^{2} x \cos ^{2} y+\phi_{v} \cos ^{2} x=0$

We determine the following system of equations from the constraints obtained above in the table of monomial coefficients.

$$
\begin{gathered}
\text { (a.) } \xi=\xi(y, v),(b .) \xi_{y}=-\phi_{x} \cos ^{2} x,(c .) \xi_{v}=-\eta_{x} \cos ^{2} x \cos ^{2} y,(d .) \xi=\phi_{y} \cot x, \\
\text { (e.) }-\xi \sin x \cos y-\phi \cos x \sin y+\eta_{v} \cos x \cos y=0,(f .) \eta_{y} \cos ^{2} y+\phi_{v}=0 .
\end{gathered}
$$

From (a.), we have $\xi$ to be a function of $y$ and $v$ alone.

From (b.), we have $\xi_{y y}=-\phi_{x y} \cos ^{2} x$, and from (d.),

$$
\phi_{x y} \cos ^{2} x=\xi
$$

by differentiating partially with respect to $x$. Upon comparison with (b.),

$\xi_{y y}=\xi$. Hence, we determine that $\xi=\alpha(v) \cos y+\beta(v) \sin y$.

Substituting this back in $(d),. \quad \phi=\tan x[\alpha(v) \sin y-\beta(v) \cos y]+\gamma(v, x)$.

From (c.), we have

$$
\begin{gathered}
\alpha^{\prime}(v) \cos y+\beta^{\prime}(v) \sin y=-\eta_{x} \cos ^{2} x \cos ^{2} y \Leftrightarrow \\
\eta=-\alpha^{\prime}(v) \sec y \tan x-\beta^{\prime}(v) \tan y \sec y \tan x+\delta(y, v) .
\end{gathered}
$$

Upon substitution of the above obtained expressions for $\phi$ and $\eta$ in $(f$.), we realize that

$$
-2 \beta^{\prime}(v) \sec y \tan x+\delta_{y} \cos ^{2} y+\gamma_{v}=0
$$

after simplification. Substituting the expressions obtained for $\xi, \eta$ and $\phi$ in (e.), we realize that

$$
\left(-\alpha(v)-\alpha^{\prime \prime}(v)-\beta^{\prime \prime}(v) \tan y\right) \tan x-\gamma \sin y+\delta_{v} \cos y=0 .
$$

We shall take $\left\{k_{j}\right\}_{j \in \mathbb{N}}$ to be arbitrary constants in what ensues. Feasible deductions from the above equation are the following:

(I.) $\alpha(v)+\alpha^{\prime \prime}(v)=0 \Leftrightarrow \alpha(v)=k_{1} \cos v+k_{2} \sin v$

(II.) $\beta(v)=k_{3}$

(III.) $\delta_{v}=\gamma \tan y$

(IV.) $\gamma=\gamma(v)$

As such, from (III.) and (IV.) we have $\delta_{y}=\kappa(v) \sec ^{2} y$, where $\kappa=\int \gamma(v) d v$. Substituting these values in the modified equation for $(f$.), we realize

$$
\int \gamma(v) d v+\gamma_{v}=0 \Longrightarrow \gamma(v)+\gamma_{v v}=0 \Longrightarrow \gamma(v)=k_{4} \cos v+k_{5} \sin v .
$$

The final variable needed to be determined is $\delta$ :

$$
\delta=\int \gamma d v \tan y=\left[k_{4} \sin v-k_{5} \cos v\right] \tan y .
$$


Upon determining solutions to this system, we observe a general accommodated infinitesimal symmetry of the system of O.D.E's for the glome's geodesics via the hyperspherical coordinate system:

$\mathbf{v}=\left[\left(k_{1} \cos v+k_{2} \sin v\right) \cos y+k_{3} \sin y\right] \frac{\partial}{\partial x}$

$+\left[\left(k_{1} \cos v+k_{2} \sin v\right) \tan x \sin y-k_{3} \tan x \cos y+k_{4} \cos v+k_{5} \sin v\right] \frac{\partial}{\partial y}$

$+\left[\left(k_{1} \sin v-k_{2} \cos v\right) \tan x \sec y+\left(k_{4} \sin v-k_{5} \cos v\right) \tan y\right] \frac{\partial}{\partial v}$.

We can then separate this general symmetry into one-parameter symmetries by the five constants $\left\{k_{i}\right\}_{i=1}^{5}$ as follows -

$$
\sum_{i=1}^{5} k_{i} \chi_{i}
$$

By computing the Lie brackets of these accommodated single parameter subgroups, one can then determine stability of the infinitesimal symmetry system, and determine the subgroups of the overall admitted invariance symmetry group of the Lagrangian in question. A sixth accommodated infinitesimal symmetry is actually revealed in this process, being the trivial translation one-parameter group $\left[\chi_{6}=\frac{\partial}{\partial v}\right]$. Computation of Lie brackets is particularly instrumental in reducing the associated Euler-Lagrange ordinary differential equation system, by exposing the right invariants to be employed together. The element in the $i$ 'th row and $j$ 'th column of the table of Lie brackets below is the vector field $\left[\chi_{i}, \chi_{j}\right]$.

Note that the characterization of Lie brackets

$$
[X, Y] f=X(Y(f))-Y(X(f))
$$

for $X=X^{i} \frac{\partial}{\partial x_{i}}, Y=Y^{j} \frac{\partial}{\partial x_{j}}$ and any $C^{\infty}$ function $f$ gives us the formula

$$
[X, Y]=\sum_{i} \sum_{j}\left\{X^{j} \frac{\partial Y^{i}}{\partial x_{j}}-Y^{j} \frac{\partial X^{i}}{\partial x_{j}}\right\} \frac{\partial}{\partial x_{i}} .
$$

\begin{tabular}{|c|cccccc|}
\hline & $\chi_{1}$ & $\chi_{2}$ & $\chi_{3}$ & $\chi_{4}$ & $\chi_{5}$ & $\chi_{6}$ \\
\hline$\chi_{1}$ & 0 & $-\chi_{6}$ & $-\chi_{4}$ & $\chi_{3}$ & 0 & $\chi_{2}$ \\
$\chi_{2}$ & $\chi_{6}$ & 0 & $-\chi_{5}$ & 0 & $\chi_{3}$ & $-\chi_{1}$ \\
$\chi_{3}$ & $\chi_{4}$ & $\chi_{5}$ & 0 & $-\chi_{1}$ & $-\chi_{2}$ & 0 \\
$\chi_{4}$ & $-\chi_{3}$ & 0 & $\chi_{1}$ & 0 & $-\chi_{6}$ & $\chi_{5}$ \\
$\chi_{5}$ & 0 & $-\chi_{3}$ & $\chi_{2}$ & $\chi_{6}$ & 0 & $-\chi_{4}$ \\
$\chi_{6}$ & $-\chi_{2}$ & $\chi_{1}$ & 0 & $-\chi_{5}$ & $\chi_{4}$ & 0 \\
\hline
\end{tabular}

We hereby ascertain stability of the accommodated Lie group of infinitesimal symmetries for the formulated geodesic variational problem, with four subgroups listed below.
(a.) $\left\{\chi_{1}, \chi_{2}, \chi_{6}\right\}$
(b.) $\left\{\chi_{1}, \chi_{3}, \chi_{4}\right\}$
(c.) $\left\{\chi_{4}, \chi_{5}, \chi_{6}\right\}$
(d.) $\left\{\chi_{2}, \chi_{3}, \chi_{5}\right\}$

A significant prospective benefit of the computation done here is an avenue for utilisation of joint invariants of any subgroup of the differential equation system in an effort to simplify the simultaneous pair of O.D.E's determined via the Lagrangian. However, in this body of work, we shall make direct reference to the collapsed equation in (Opara, 2017, pp.10):

$\dot{y} \sin x \cos y\left(k-2 \cos ^{2} x \cos ^{2} y\right)+\ddot{y} \cos x \cos y\left(\cos ^{2} x \cos ^{2} y-k\right)+k \sec x \sin y+k(\dot{y})^{2} \cos x \sin y-(\dot{y})^{3} \cos ^{4} x \sin x \cos ^{3} y=$ $0 \quad \cdots(E=0)$

derived from substituting the outcome of one Euler-Lagrange equation:

$$
\Lambda_{v}-\frac{d}{d x}\left(\Lambda_{\dot{v}}\right)=0
$$


in the formulation of the second equation in the pair:

$$
\Lambda_{y}-\frac{d}{d x}\left(\Lambda_{\dot{y}}\right)=0
$$

For emphasis, the constant $k$ in the O.D.E above is in the interval $[0,1]$ and an overset dot connotes differentiation with respect to the independent variable $x$ of the system. The reader may confirm that $(E=0)$ accommodates the oneparameter subgroup $\left[\chi_{3}=\sin y \frac{\partial}{\partial x}-\cos y \tan x \frac{\partial}{\partial y}\right]$ computed earlier, since $\left[\operatorname{pr}^{(2)} \chi_{3}(E)=0\right]$ whenever $[E=0]$. Reckoning with the common benefit of knowledge of an accommodated one-parameter symmetry of an O.D.E, we have foresight that the equation $[E=0]$ of the second order would be reduced to a significantly simpler equation of lower order, upon replacement of the variables $x$ and $y$ with the canonical coordinates of the symmetry $\chi_{3}$. Let the canonical coordinates of this symmetry be $(\tau, \omega)$, whereby $\omega$ is the invariant and $\tau$ the other canonical coordinate. To determine $\omega$, we solve the equation

$$
\frac{d x}{\sin y}=\frac{d y}{-\tan x \cos y},
$$

revealing that

$$
\cos x \cdot \cos y=[\text { constant }] \text {. }
$$

This means we may take $\omega$ to be $\cos x \cos y$, or any smooth function of this term. Moreover,

$$
\tau=\int \frac{d x}{\sin y}
$$

whereby $y$ is expressed in terms of $x$ and $\omega$ (using $\omega=\cos x \cos y$ ), and $\omega$ is momentarily taken as a constant when evaluating this integral (Hydon, 2000, pp.24). Thus, we obtain $\tau(x, y)=\arctan (\cot x \sin y)$ as the second canonical coordinate.

Besides the infinitesimal form of a one-parameter symmetry, the global form also reveals other details present. The global form $(X(x, y, \lambda), Y(x, y, \lambda))$ is determined by integrating the autonomous O.D.E system:

$$
\frac{d X}{d \lambda}=\sin Y, \frac{d Y}{d \lambda}=-\tan X \cos Y
$$

subject to the initial constraints $\left.(X, Y)\right|_{\lambda=0}=(x, y)$, whereby $x$ and $y$ are temporarily taken as constants in the course of integration. The solutions obtained here are

$$
X=\arcsin [\sin x \cos \lambda-\cos x \sin y \sin \lambda] \quad \text { and } \quad Y=\arctan [\tan y \cos \lambda+\tan x \sec y \sin \lambda] .
$$

These functions satisfy the customary requirements: $\omega(X, Y)=\omega(x, y)$ and $\tau(X, Y)=\tau(x, y)+\lambda$.

\section{Discussion}

The equation $[E=0]$ in focus becomes significantly simpler upon replacement of the initial variables $(x, y)$ with the canonical coordinates $(\tau, \omega)$ of the accommodated symmetry we have engaged. Specifically, this equation impressively reduces to the first order representation:

$$
\omega^{\prime}(\tau)=\left(1-\omega^{2}\right) \tan \left[ \pm \arccos \sqrt{\frac{\alpha-\left(\omega^{2} \cos ^{2} \tau+\sin ^{2} \tau\right)}{\left(\frac{k}{\omega^{2}}-1\right)\left(\omega^{2} \cos ^{2} \tau+\sin ^{2} \tau\right)}}+\arctan \left(\frac{\omega}{\tan \tau}\right)\right] .
$$

Another constant $\alpha$ of integration in the first order O.D.E above comes about at a stage of the simplification process from the prior form $[E=0]$.

A comparison of this result to what holds for the geodesic equation of the simpler 2-sphere $\left(S^{2}\right)$ reveals some interesting connections. This sphere is obtained as the intersection of the glome $S^{3}$ with the hyperplane $[v \equiv 0]$. Capturing this manifold with the same spherical coordinate system used above for $S^{3}$ yields the geodesic differential equation:

$$
\frac{d^{2} y}{d x^{2}}=2 \frac{d y}{d x} \tan x+\left(\frac{d y}{d x}\right)^{3} \sin x \cos x .
$$

This equation also accommodates the symmetry $\left[\chi_{3}=\sin y \frac{\partial}{\partial x}-\cos y \tan x \frac{\partial}{\partial y}\right]$, which was used to simplify the geodesic equation for the glome. It may be argued that this inherited symmetry of the geodesic equations is based on the fact that $S^{2}$ 
is a totally geodesic hypersurface of $S^{3}$. Simply put, all geodesics of the submanifold $S^{2}$ are also geodesics of the glome, which apparently corroborates the shared symmetry of their geodesic differential equations. Determination of totally geodesic hypersurfaces invariably simplifies any theoretical and computational considerations from closed Riemannian manifolds.

In general, the geodesic equation for $\left(S^{2}\right)$ admits the 3-dimensional infinitesimal rotation group on Euclidean 3-space (Stephani, 1989, pp.78), which could contribute otherwise to reduction via invariance transformations for the equation $[E=0]$ we have engaged. Because none of the admitted symmetries was used in collapsing the required pair of EulerLagrange equations to obtain $[E=0]$, the resulting equation may retain invariance under those infinitesimal symmetries in terms of the variables $x$ and $y$. As a major part of what has been established in the previous section, the geodesic equations for $\left(S^{3}\right)$ admit the 6-dimensional infinitesimal rotation group $\left(\mathrm{SO}_{4}\right)$ on Euclidean 4-space. Hence, there apparently remains a number of significant consequential properties to be harnessed from this identified symmetry group classification, considering also their explicit functional expressions herein made available.

\section{Conclusion}

Geodesics constitute a class of minimal submanifolds of closed 3-manifolds. Another class of minimal submanifolds is that of minimal hypersurfaces. This latter class of 2-dimensional minimal submanifolds are particularly well suited to the study of intrinsic properties of their ambient manifolds in static or evolutionary states (Anciaux, 2011, pp.18 - 27). As a matter of definite interest, minimal hypersurfaces have been explored severally in academic archives to elucidate details of manifolds' evolution in course of the Ricci Flow. In the weak formulation of Partial Differential Equations (P.D.E's) for stable minimal hypersurfaces, it is relevant to attempt identification of conservation laws in action. In this way, Noether's theorem becomes a guarantor of simplification of the associated P.D.E's by accommodated symmetries.

Besides probing the inner geometry of 3-manifolds using minimal hypersurfaces, the solitons of Ricci Flow are characterized as equilibrium states of the metrics in the diffusion-reaction equations in process (Cao et al., 2003, pp.4). This again gives rise to associated conservation laws and an invaluable avenue to engage Noether's theorem. Since its discovery, this theorem has been at the hub of simplification achievements for systems of differential equations, but the chosen vantage point of the glome has specifically been seen to conceal a plethora of theoretical wealth in this regard.

\section{References}

Anciaux, H. (2011). Minimal Submanifolds in Pseudo-Riemannian Geometry. World Scientific Publishing Co. Pte. Ltd. https://doi.org/10.1142/7542

Brunt, B. (2004). The Calculus of Variations. Springer.

Cao, H. D., Chow, B., Chu, S. C., \& Yau, S. T. (2003). Collected Papers on the Ricci Flow. International Press.

Dresner, L. (1999). Applications of Lie's Theory of Ordinary and Partial Differential Equations. IOP Publishing Limited. https://doi.org/10.1887/0750305312

Hydon, P. E. (2000). Symmetry Methods for Differential Equations - A Beginner's Guide. Cambridge University Press. https://doi.org/10.1887/0750305312

Olver, P. J. (1993). Applications of Lie Groups to Differential Equations (Second Edition). Springer - Verlag New York, Inc. https://doi.org/10.1007/978-1-4612-4350-2

Opara, U. (2017). Riemannian Geodesics: An Illustration From the Calculus of Variations. Cornell University e-print Archive - arXiV:1703.06430v1 [math.GM].

Stephani, H. (1989). Differential Equations: Their Solution Using Symmetries. Cambridge University Press. https://doi.org/10.1017/CBO9780511599941

\section{Copyrights}

Copyright for this article is retained by the author(s), with first publication rights granted to the journal.

This is an open-access article distributed under the terms and conditions of the Creative Commons Attribution license (http://creativecommons.org/licenses/by/4.0/). 